\title{
The regurgitant bicuspid aortic valve: Recalibrating our surgical strategies
}

\author{
James K. Kirklin, MD
}

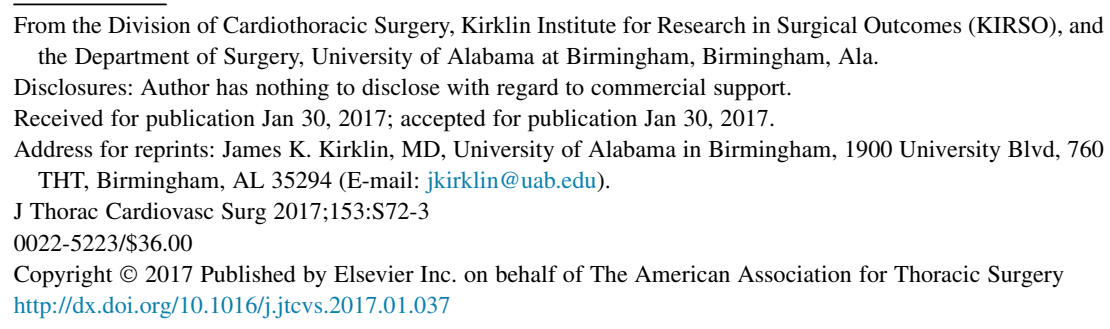

The prevalence of a bicuspid aortic valve in the general population and its importance as a cause of aortic regurgitation warrant careful reading of the analysis by Schneider and colleagues. ${ }^{1}$ The frequently associated aortopathy and at least moderate dilation of the aortic root has generated controversy regarding optimal surgical therapy for regurgitation of a bicuspid aortic valve. This report analyzes a 20year experience with more than 350 patients by Schäfers and colleagues ${ }^{2}$ with a relatively uniform surgical strategy for bicuspid aortic valve regurgitation that combines aortic valve repair with aortic root remodeling. Their propitious outcomes, including a very low hospital mortality, nearly $80 \%$ freedom from reoperation at 15 years, and generally stable repairs, are a testament to their expertise with this malformation. The relatively young age (mean 49 years) of this patient cohort highlights the appeal of reconstructive surgery rather than possibly 2 to 3 decades of exposure to chronic anticoagulation with a mechanical valve as part of an aortic root replacement. For the surgeon wishing to refine or expand techniques of aortic valve reconstruction in this setting, the identification of calcific plaques extending beyond the raphe area and the need for pericardial patch reconstruction as risk factors for valve failure provide guidance for situations to avoid aortic valve reconstruction.

When viewing these outstanding results in the context of methods used by many experienced cardiac surgeons, several cautionary notes seem in order. Of importance is the remodeling technique of valve-sparing aortic root replacement, which appears to have been the routine method of root reconstruction during this 20-year experience. ${ }^{2}$ Many (and perhaps most) surgeons use the reimplantation technique for valve-sparing aortic root replacement, which may complicate complete adoption of the described technique of aortic valve repair, including the recommendation for nearly routine circumferential suture annuloplasty.

The finding that $95 \%$ of patients appear to have trivial or no aortic regurgitation at hospital discharge ${ }^{1}$ demonstrates the effectiveness of their technique. Their relative infrequency of late progression of aortic insufficiency after repair

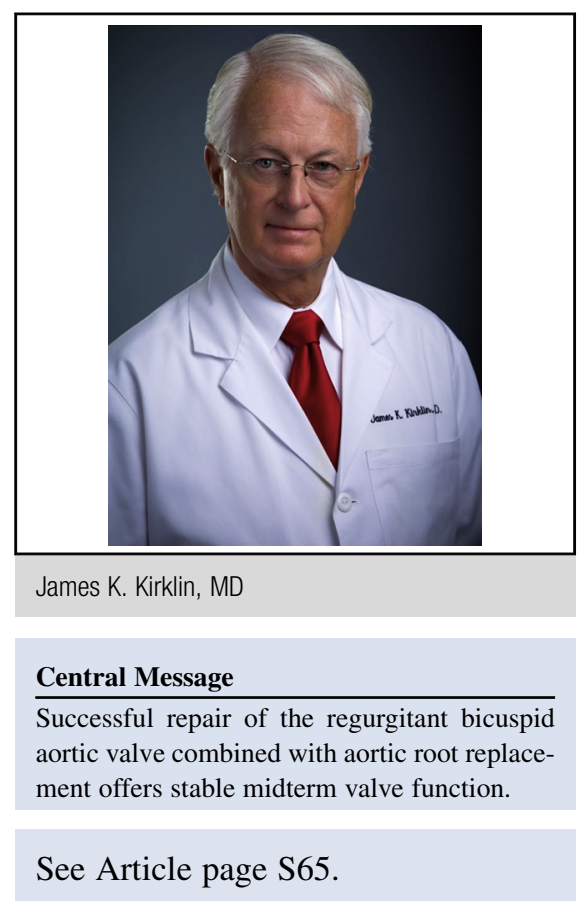

of a bicuspid aortic valve contrasts with some other reports. One is left wondering whether these exceptional results stem from their huge ongoing experience with bicuspid aortic valve reconstruction or whether their technique of aortic root reconstruction is playing a major role. Whether this translates into the wisdom of accepting grade 2 to 3 aortic insufficiency as sufficient improvement on grade 5 aortic insufficiency at the end of the operation (assuming that it will likely remain stable) remains unresolved.

Finally, it will be interesting to follow the impact of transcatheter aortic valve replacement on decisions regarding valve repair versus replacement. Many surgeons are already lowering their threshold for nonmechanical valve options in aortic valve surgery with the expectation that transcatheter aortic valve replacement will become a mature option for secondary aortic valve replacement when a bioprosthetic valve or valve repair fails. Valve-sparing root replacement combined with reconstruction of the regurgitant bicuspid aortic valve thus becomes even more relevant in our cardiac surgical practice. Given the option of (or patient expectation for) a possible subsequent transcatheter aortic valve replacement, the application of techniques for a good even if not perfect repair of a bicuspid aortic valve combined with aortic root replacement may well become a necessary part of the skilled adult cardiac surgeon's armamentarium. 


\section{References}

1. Schneider U, Feldner SK, Hofmann C, Schöpe J, Wagenpfeil S, Giebels C, et al. Two decades of experience with root remodeling and valve repair for bicuspid aortic valves. J Thorac Cardiovasc Surg. 2017; 153:S65-71
2. Schäfers HJ, Langer F, Aicher D, Graeter TP, Wendler O. Remodeling of the aortic root and reconstruction of the bicuspid aortic valve. Ann Thorac Surg. 2000;70:542-6.

3. Casselman FP, Gillinov AM, Akhrass R, Kasirajan V, Blackstone EH Cosgrove DM. Intermediate-term durability of bicuspid aortic valve repair for prolapsing leaflet. Eur J Cardiothorac Surg. 1999;15:302-8. 